\title{
Does an Actomyosin System Supplement the Axoneme in Mammalian Sperm
}

\author{
Terry Perot, Ayele Gugssa, William Eckberg, Balwant Ahluwalia (Corresponding author) \\ Baccio Baccetti \& Winston Anderson \\ Department of Biology and Obstetrics \& Gynecology \\ Howard University Washington D.C. 20059 \\ University of Sienna, Siena, Italy
}

Tel:202-865-1662Ｅ-mail: bahluwalia@howard.edu

\begin{abstract}
Boar sperm fixed in paraformaldehyde, pemeabilized in cold acetone and incubated in the presence of DAPI and Rhodamine-conjugated phalloidin or with fluorescein-conjugated cytochalasin D show brilliant blue staining of the nucleus and brilliant red or green fluorescence respectively in the middle piece and tail regions of the sperm. The results suggest the presence of F-actin in the boar sperm middle piece. Immunocytochemical studies also show the presence of tropomyosin in the middle piece region of boar sperm.

Western Blot studies, using lysates of isolated boar sperm middle pieces and tails, indicate prominent sperm-actin bands with similar electrophoretic patterns of rat cardiac and striated muscle. The results strongly suggest that a $41 \mathrm{kDa}$ actin, similar to cardiac or striated muscle actin, is present in sperm middle pieces and corroborates the phalloidin and cytochalasin D localization by fluorescence microscopy.

We suggest that the simple sliding filament mechanism of the axoneme may not, by itself, impose the propulsive forces needed to propel large spermatozoa through the viscous prefertilization milieu. We further propose that an actomyosin system associated with the microtubule/dynein complex might play a role in sustained motility of mammalian spermatozoa.
\end{abstract}

Keywords: Actomyosin, Axoneme, Flagella, Boar sperm

\section{Introduction}

Analysis of the motion of flagella and cilia would suggest a mechanism based on the sliding of microtubules (1-2). They may be sliding in relation to each other, and the sliding out of a tubule preferentially on one side of the cilium could produce a bending in that direction (3). Thus the dynein cross bridges or motors that form connections with microtubules are implicated in this movement. Presumably, a dynein complex attached to the A tubule of a doublet is in a position to interact with the B tubule of an adjacent complex. Studies with isolated Tetrahymena cilia suggest that free dynein arms are polarized and tilt uniformly away from the direction of active sliding towards the base of the cilium (2). More recent studies confirm dynein-tubulin interactions for microtubule-based motility (4). In all cases ATP hydrolysis appears to be involved in motion. Thus the sliding-filament/microtubule hypothesis of ciliary motility, generated by ATP, is considered acceptable dogma for single cells, including simple sperm, and ciliated epithelia. In these cells, microtubular structures are arranged linearly, and thus impose a vectoral displacement of matter in an aqueous medium. The velocity of sliding is directly related to the propulsive force generated in an aqueous environment and is proportionately related to the mass of the organism.

What happens when a massive unicellular organism, equipped with a single cilium or flagellum, must move in a viscous medium? Structurally, filaments and fibers that are accessory to the microtubule/dynein complexes are present in some of these large cells. A case in point is sperm of most mammals that show the presence of accessory coarse fibers and a plethora of axonemal filaments and fibers (5). Biochemically, isolated coarse fibers appear not to contain contractile proteins and might just represent stiffening rods in the middle pieces and tails of large sperm. Might these coarse fibers represent attachment sites for contractile proteins also located in the axoneme? Conceivably, an ATP-regulated contractile system accessory to the microtubule/dynein system may be essential to generate the motive and propulsive forces needed to propel large cumbersome sperm cells in a viscous milieu. 
We suggest that the simple sliding filament mechanism of the axoneme may not, by itself, impose the propulsive forces needed to propel large spermatozoa through the viscous prefertilization milieu. We further propose to establish that an actomyosin system associated with the microtubule/dynein complex might play a role in sustained motility of mammalian spermatozoa.

\section{Materials and Methods}

Fresh boar semen was purchased commercially from the Zierke Company. Sperm were checked for motility and viability and maintained at $26^{\circ} \mathrm{C}$, prior to further use. Sperm maintained viability up to 7 days under these conditions.

\subsection{Sperm isolation and purification}

To remove the seminal fluid from sperm, semen was diluted in two changes of PBS-PMSF and centrifuged at $3000 \mathrm{~g}$ for 20 min in a refrigerated swinging bucket rotor. The pellet was resuspended and layered over a $35 \%$ and $70 \%$ Percoll/PBS gradient in a $15 \mathrm{ml}$ conical centrifuge tube and centrifuged at $2000 \mathrm{x}$ g for $5 \mathrm{~min}$ followed by centrifugation at $4000 \mathrm{x} \mathrm{g}$ for $10 \mathrm{~min}$. The fluffy mass of sperm near the middle of the $70 \%$ Percoll layer was aspirated using a pipette and transferred to a centrifuge tube. The collected sperm were resuspended in PBS, washed, and pelleted by centrifugation.

\subsection{Sperm tail isolation}

Spermatozoa suspended in PBS-PSMF were homogenized at 30 second intervals until the head was separated. Complete separation of sperm head and tail was checked by microscopy. The decapitated spermatozoa were washed in PBS-PSMF and centrifuged for $10 \mathrm{~min}$. The pellet was resuspended in $20 \%(\mathrm{w} / \mathrm{v})$ sucrose in PBS-PMSF before layering over a $20 \%, 40 \%$ and $60 \%$ sucrose gradient and centrifuged at $3000 \mathrm{x}$ g for $90 \mathrm{~min}$ in a refrigerated swinging bucket rotor. Sperm tail fractions were collected from the $40-60 \%$ sucrose interface and purity was assessed by microscopy. The sperm tail fraction was re-suspended in $20 \%(\mathrm{w} / \mathrm{v})$ sucrose in PBS-PMSF and centrifuged again at $3000 \mathrm{x}$ g through a $20 \%, 40 \%$ and $60 \%$ sucrose step gradient for $60 \mathrm{~min}$. Sperm tails were collected from $40-60 \%$ interface, which contained $<1 \%$ sperm head contamination. The sperm tails were subsequently diluted with PBS-PMSF and pelleted by centrifugation at $15000 \mathrm{x}$ g for $10 \mathrm{~min}$.

\subsection{Western Blot Analysis for Actin}

For detection of actin, purified sperm tails, rat striated and cardiac muscle were lysed in 3 different lysis buffers (a) ice-cold NP-40 lysis buffer (50 mM Tris-HCl, pH 7.5, $150 \mathrm{mM} \mathrm{NaCl}, 0.5 \% \mathrm{NP}-40$, and $50 \mathrm{mM} \mathrm{NaF}$ ), (b) $0.1 \mathrm{M}$ Tris $-\mathrm{HCL}, 1 \mathrm{M}$ EDTA, $8 \mathrm{M}$ urea and $0.05 \mathrm{M}$ DDT and (c) $100 \mathrm{mM}$ Tris-HCL, $100 \mathrm{mM} \mathrm{KCl}, 200 \mathrm{UM}$ EDTA, and $1.5 \mathrm{mM}$ $\mathrm{MgCl} 2$.The resulting lysates were centrifuged at $14000 \mathrm{xg}$ for $10 \mathrm{~min}$ to remove cell debris. The samples were mixed with SDS sample buffer and boiled for 5 min. $20 \mu \mathrm{l}$ of the prepared lysates, as well as purified actin (Abcam, Cambridge, Ma) were resolved on 12\% SDS-polyacrylamide gels and electroblotted to PVDF membrane (Hybond, Amersham). Membranes were blocked for $1 \mathrm{hr}$ at room temperature with 5\% nonfat dry milk in PBS containing $0.05 \%$ Tween 20 and incubated with antibodies specific to actin $(0.5 \mu \mathrm{g} / \mathrm{mL}$, Molecular probes, Eugene Oregon, USA) for 24 $\mathrm{hr}$ in the same buffer. After washing, the blots were incubated for $1 \mathrm{hr}$ with a 1:4000 dilution of horseradish peroxidase (HRP)-conjugated secondary antibody and visualized using an enhanced chemiluminescence detection system (Amersham).

\subsection{Localization of Actin by Phalloidin and Cytochalasin}

Sperm were resuspended with PBS-PMSF and washed three times before pelleting by centrifugation and fixed for 30 min with a $4 \%$ paraformaldehyde buffered with PBS or sodium cacodylate. The sperm samples were rinsed overnight with several changes of PBS and then permeabilized with cold acetone $\left(-20^{\circ} \mathrm{C}\right)$ for $5-10 \mathrm{~min}$. Cells were washed and incubated for $1 \mathrm{hr}$ in a 1:1000 dilution of Texas Red, Alexa, or Fluorescein phalloidin (Invitrogen, Molecular Probes) in PBS. The cells were washed three times, counterstained with DAPI for 3 min and washed several times with PBS before mounting in Slow Fade. Some sperm were fixed, washed and permeabilized as above and incubated in fluorescein-conjugated cytochalasin D (Invitrogen, Molecular Probes). The samples were viewed under a Zeiss fluorescence microscope using a Rhodamine filter (wavelength $550 \mathrm{~nm}$ ), or a fluorescent triple excitation filter for UV, Rhodamine and Fluorescein.

\subsection{Immunocytochemical Procedres}

Sperm, washed of seminal fluid, were fixed for $30 \mathrm{~min}$ in $4 \%$ buffered paraformaldehyde. The fixed samples were washed thoroughly with PBS and permeabilized with cold acetone $\left(-20^{\circ} \mathrm{C}\right)$ for $5 \mathrm{~min}$. Sperm were washed with PBS then blocked with 1\% Bovine serum albumin for $30 \mathrm{~min}$, washed and incubated in a 1:500 dilution of primary antibody to tropomyosin (Abcam, Cambridge, MA.) for 1 hour except the control which was incubated with PBS. The samples were washed three times with PBS then incubated with FITC or Rhodamine conjugated secondary antibody (1:500 dilution) for $30 \mathrm{~min}$. The samples were washed three times with PBS and counterstained with diluted DAPI, washed and mounted in Slow Fade and visualized under a Zeiss fluorescence microscope. 


\section{Results}

Sperm fixed in paraformaldehyde, pemeabilized in cold acetone and incubated in the presence of DAPI and Rhodamine conjugated phalloidin show brilliant blue staining of the nucleus and brilliant red middle pieces (Fig. 1). The phalloidin reaction started just below the neck region of the sperm, passed through the middle piece region and extended into the sperm tail (Fig. 2). To verify the presence of actin, sperm that were fixed in paraformaldehyde, permeabilized with acetone and incubated with fluorescein-conjugated cytochalasin D, showed similar green fluorescence in the middle piece and tail regions. The results confirm the presence of F-actin in the boar sperm middle piece. When reacted with fluorescein-conjugated mitotracker and phalloidin, faint staining of the actin was observed. The presence of the surrounding mitochondria of the middle piece obscures actin staining in the axoneme.

To confirm the presence of actin in boar sperm middle pieces, sperm tails were lysed with several buffers specific for mammalian cell actin isolation and electrophoresed to immunodetect actin. Sperm tail lysates were electrophoresed alongside rat cardiac and striated muscle lysates and reacted to reveal actin. Western blot results revealed actin in sperm, striated muscle and cardiac muscle (Fig. 5). These actin bands had identical molecular weights around 41KDa.

Sperm fixed in paraformaldehyde, permeabilized with acetone, washed and incubated with anti-tropomyosin antibodies reveal a strong red fluorescence reaction after treatment with a Fluorescein-conjugated secondary antibody to antitropomyosin (Fig. 6). The results suggest a possible actin-tropomyosin binding site in the middle pieces and tails of the boar sperm.

\section{Discussion}

The phallotoxins are reliable probes for labeling, identifying and quantitating F-actin in formaldehyde-fixed and permeabilized tissues and cells. Conjugated to green or red fluorescent dyes, fluorescein (FITC) or rhodamine (RITC) these probes have been used to accurately visualize actin filament assemblies, cytoskeletal reorganizations, and actin-related movement of intracellular organelles, and cell movements (6-13) Studies with cytochalasins B and D conclusively indicate that this cell-permeant fungal toxin binds to the barbed end of actin filaments preventing association of dissociation of subunits. Green or orange-fluorescent cytochalasin D (BODIPY FL; BODIPY TMR) are accurate indicators of F-actin in formaldehyde and permeabilized tissues and cells (Invitrogen, Molecular Probes). The literature is replete with examples showing the consistency of fluorescent-phalloidin and cytochalasin D probes for actin in a broad variety of cells (14-18). Our results consistently show the presence of actin from the neck to tail regions of formaldehyde-fixed and permeabilized boar spermatozoa. Previous studies have used electron microscopy to localize filaments, presumably actin, in cytosols of invertebrate spermatozoa and it has been speculated that thin axonemal filaments associated with microtubules may have actomyosin properties.

Western Blot studies, using lysates of isolated boar sperm middle pieces and tails, indicate prominent sperm-actin bands with similar electrophoretic patterns of rat cardiac and striated muscle. The results strongly suggest that a $41 \mathrm{kDa}$ actin, similar to cardiac or striated muscle actin, is present in sperm middle pieces and corroborates the phalloidin and cytochalasin D localization by fluorescence microscopy.

How much and what role axonemal actin plays in sperm motility is not yet determined, as is their localization with respect to the axonemal microtubules and coarse fibers, and spermatozoan motility. Isolated coarse fibers show no actin reactivity. It might be construed that the coarse fibers, that seem keratin-like in nature, may represent binding sites for an active actmyosin system. The perimicrotubular filaments in close proximity to the axonemal microtubules and coarse fibers might represent an accessory actomyosin complex which together with the microtubular system generates the motive forces for spermatozoan propulsion, especially in a viscous internal fertilization environment.

By binding specifically to actin, tropomyosin plays a central role in the regulation of actonmyosin contractile systems (19-20). Immunocytochemical studies show the presence of tropomyosin in the middle piece region of boar sperm. Conceivably, actin-tropomyosin conjugation might represent a functional actomyosin complex needed for motility.

Papers by Fawcett (5) and others clearly reveal the presence of accessory filaments and fibers adjacent to the axonemal micotubular elements of the sperm tail. The filamentous configurations are especially prominent in sperm of the 13-lined ground squirrel and other species (5)). Less dramatic filamentous assemblies occupy the axonemal region of the middle piece of other mammalian sperm and are not prominent in sperm shed in an aqueous, non-viscous environment. Indeed, filamentous assemblies are not prominent in boar sperm, but if we are to believe the images after phalloidin and cytochalasin treatment the conclusion can be made to support the presence of actin at these sites. Incubation of sperm in $\mathrm{DAB} / \mathrm{OsO} 4$ solutions reveals the existence of a matrix between the coarse fibers and surrounding the axonemal microtubules (Fig. 7). The Western blot data confirm actin in the middle piece and sperm tails. Similarly, tropomyosin immunofluorescence suggests the presence of a functional actomysoin system in the middle piece region of the boar sperm. More work is needed to determine the exact structural and functional relationship between the microtubular and actomyosin filaments and their contributions to sperm motility. 


\section{References}

Bryan, J. and Wilson, L. (1971). Are cytoplasmic microtubules heteropolymers. PNAS, USA 68: 1762-1766.

Burgess, D.R. Broschat, K.O. and Hayden, J.M. (1987). Tropomyosin distinguishes between the two actin-binding sites of villin and affects actin-binding properties of other brush border proteins. J. Cell Biol., 104: 29-40.

Carlier, M.F. Criquet, P. Pantaloni, D. and Korn, E.D. (1986). Interaction of cytochalasin D with actin filaments in the presence of ADP and ATP. J. Biol Chem., 261 (5): 2041-50.

Casella, J.F., Flanagan, M.D. and Shin Lin. (1981). Cytochalasin D inhibits actin polymerization and induces depolymerization of actin filaments formed during platelet shape change. Nature, 293: 302-305.

Cooper, J.A. (1987). Effects of cytochalasin and phalloidin on actin. J. Cell Biol., 105: 1473-1478.

Eaton, B.L. (1976). Tropomyosin binding to F-actin induced by myosin heads. Science, 192:1337-1339.

Fawcett, D. W. (1970). A comparative view of sperm ultrastructure. Biol. Reprod. Supplement, 2: 90-127.

Hill, L.E. Mehegan, J.P., Butters, C.A. and Tobacman, L.S. (1992). Analysis of troponin-tropomyosin binding to actin. Troponin does not promote interactions between tropomyosin molecules. J. Biol. Chem., 267 (23):16106-13.

Koonce, M.P. and Tikhonenko, I. (2000). Functional elements within the dynein-microtubule-binding domain. Mol. Biol. Cell, 11(2): 523-529.

Lotz, M.M. and Rabinovitz, I. and Mercurio, A.M. (2000). Intestinal restitution: progression of actin cytoskeleton rearrangements and integrin function in a model of epithelial wound healing. Am. J. Path., 156:985-996.

Mortensen, K. and Larsen L.I. (2003). Effects of cytochalasin D on the actin cytoskeleton: association of neoformed actin aggregates with proteins involved in signaling and endocytosis. Cell and Mol. Life Sciences, 60:1007-1012.

Nothnagel, E., Barak, L.S. and Sanger, J. W. and Webb, W.W. (1981). Fluorescent studies on modes of cytochalasin B and phalloidin actin on cytoplasmic streaming in Chara. J. Cell Biol., 88 (2): 364-72.

Ohashi, T., Kiehart, D.P. and Erickson, H.P. (2002). Dual labeling of fibronectin matrix and actin cytoskeleton with green fluorescent protein variants. J. Cell Science, 115: 1221-1229.

Perez, R.A, Langford, G.M. Eckberg, W.R. and Anderson, W.A. (1986). Contractile proteins (actin, myosin) and tubulin are revealed within DNA-containing nucleoplasm in mature spermatozoa of Libidnia emarginata L. J. Submicroscop. Cytol. Pathol., 18: 221-248.

Pohya, Z. and Barnabas, B. (2001). Microinjected fluorescent phalloidin in vivo reveals F-actin dynamics in isolated egg cells of wheat (Triticum aestivum L) developed in situ and fertilized in vitro. J. Plant Physiol., 158 (12): $1527-1539$.

Satir, P. (1968). Studies on cilia. III. Further studies on the cilium tip and a "sliding filament" model of ciliary motility. J. Cell Biol., 39: 77-94.

Schmit, A.C. and Lambert, A.M. (1990). Microinjected fluorescent phalloidin in vivo reveals F-actin dynamics and assembly in higher plant mitotic cells. Plant Cell, 2(2):129-138.

Szczesna, D. and Lehrer, S.S. (1993). The binding of fluorecent phallotoxins to actin in myofibrils. J. Muscle Research and cell Motility, 14: 1573-2657.

Valentijn, J.A., Valentjin, K. Pastore, L.M. and Jamieson, J.D. (2000). Actin coating of secretory granules during regulated exocytosis correlates with the release of rab3D. PNAS, 97: 1091-1095.

VanBuren P., Begin, K and Warshaw, D.M. (1998.) Fluorescent phalloidin enables visualization of actin without effects on myosin's actin filament sliding velocity and hydrolytic properties in vitro. J. Mol. Cell Cardiol., 30 (2) $2777-83$.

Warner, F.D. and Mitchell, D.R. (1981). Polarity of dynein-microtubule interactions in vitro: crosslinking between parallel and antiparallel microtubules. J. Cell Biol., 89:35-44.

Westcarr, S., Farshori, P., Wyche, J.H., and Anderson, W.A. (1999). Apoptosis and differentiation in the crypt-villus unit of the rat small intestine. J. Submicroscop. Cytol Pathol., 31: 15-30.

WuBing, Fraser, D.C. and Marston, S.B. (1997). Troponin I and troponin T interact with troponin C to produce different Ca2+-dependent effects on actin-tropomyosin filament motility. Biochem. J., 327: 335-340. 

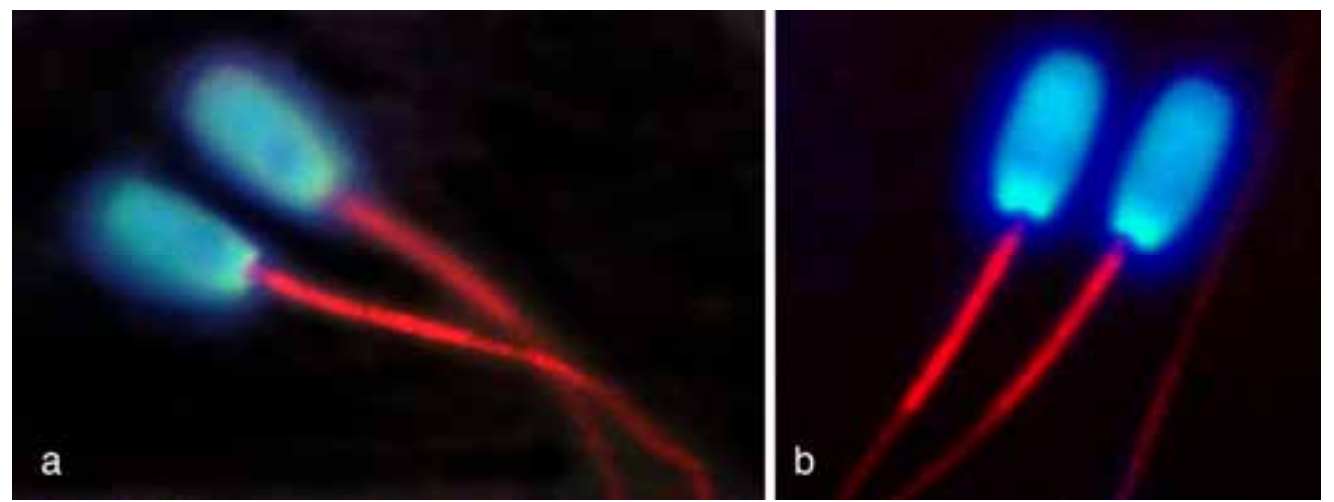

Figures 1 and 2. Texas Red-phalloidin actin is present in the middle piece and tails of paraformaldehyde-fixed and acetone permeabilized boar sperm. DAPI-reacted nuclei are also revealed. X400

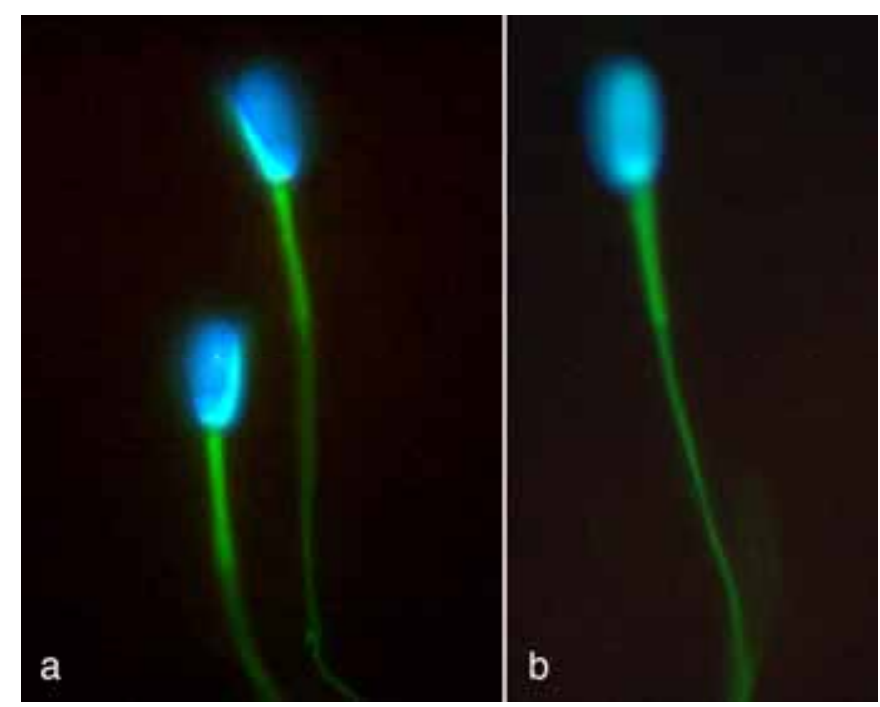

Figures 3 and 4. Fluorescein-conjugated cytochalasin D reacts with F-actin in the middle piece and sperm tails. DAPI-reacted nuclei are stained blue. X200

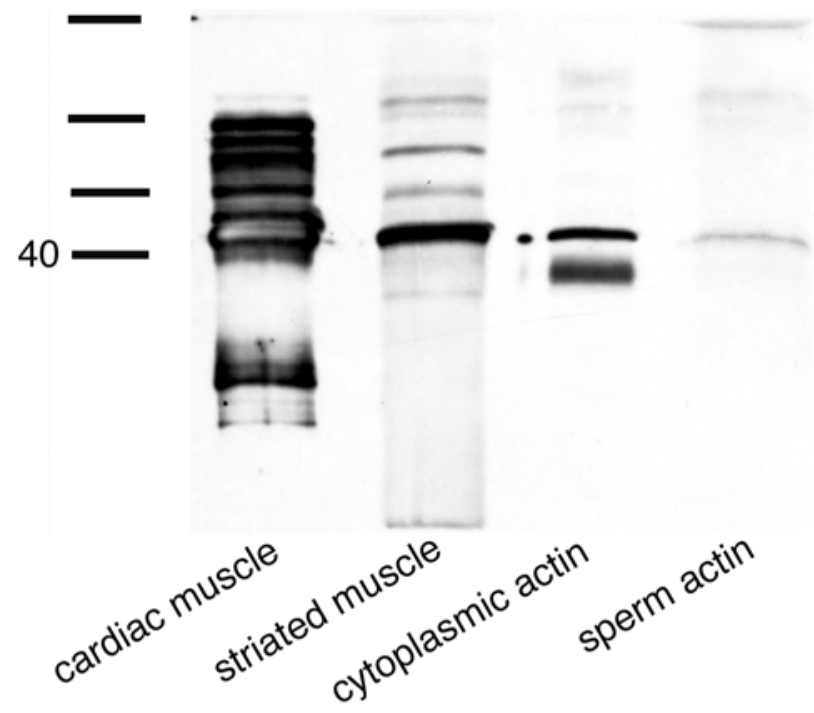

Figure 5. Western blot results revealed 41KDa actin bands cardiac (Lane 2), striated muscle (Lane 3), and in sperm lysates (Lane 5). Band 1 shows the standard and Band 4 shows pure actin (Abcam, Cambridge, MA). 


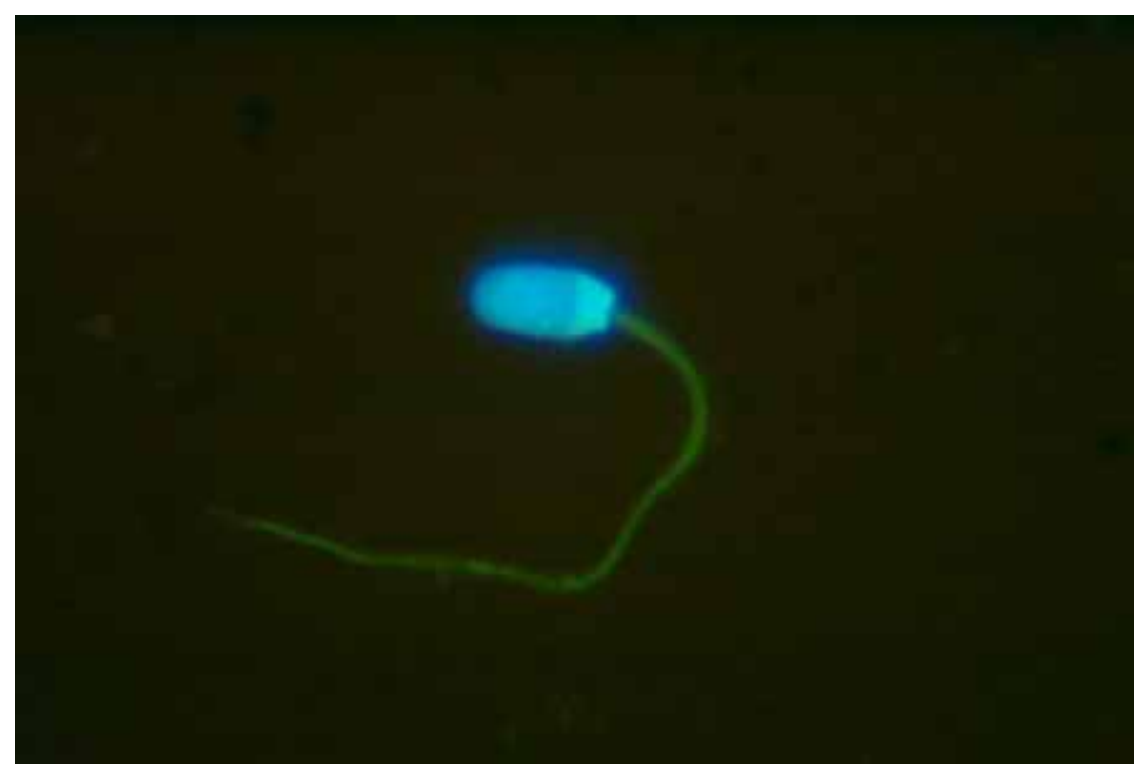

Figure 6. Immunocytochemical image reveals the presence of tropomyosin in the middle piece and tails of boar sperm. $\mathrm{X} 200$

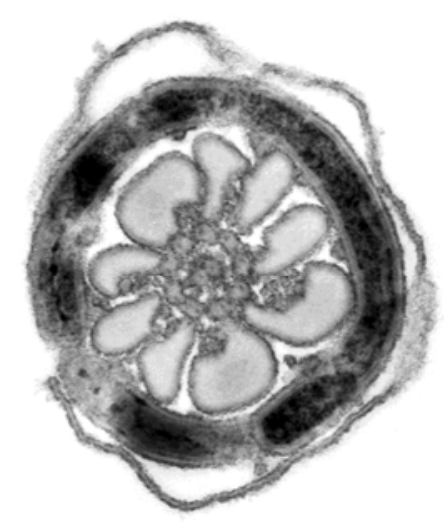

Figure 7. Sperm incubated in Diaminobenzidine and reacted with OsO4 reveal a matrix material between coarse fibers and surrounding the axonemal microtubules. X20,000 